\title{
General Relativity and Weyl Geometry
}

\author{
C. ROMERO, ${ }^{*}$ J. B. FONSECA-NETO and M. L. PUCHEU
}

January 9, 2012

\begin{abstract}
We show that the general theory of relativity may be formulated in the language of Weyl geometry. We develop the concept of Weyl frames and point out that the new mathematical formalism may lead to different pictures of the same gravitational phenomena. We show that in an arbitrary Weyl frame general relativity, which takes the form of a scalar-tensor gravitational theory, is invariant with respect to Weyl tranformations. A kew point in the development of the formalism is to build an action that is manifestly invariant with respect to Weyl transformations. When this action is expressed in terms of Riemannian geometry we find that the theory has some similarities with Brans-Dicke gravitational theory. In this scenario, the gravitational field is not described by the metric tensor only, but by a combination of both the metric and a geometrical scalar field. We illustrate this point by, firstly, discussing the Newtonian limit in an arbitrary frame, and, secondly, by examining how distinct geometrical and physical pictures of the same phenomena may arise in different frames. To give an example, we discuss the gravitational spectral shift as viewed in a general Weyl frame. We further explore the analogy of general relativity with scalar-tensor theories and show how a known Brans-Dicke vacuum solution may appear as a solution of general relativity theory when reinterpreted in a particular Weyl frame. Finally, we show that the so-called WIST gravity theories are mathematically equivalent to Brans-Dicke theory when viewed in a particular frame.
\end{abstract}

PACS numbers: 98.80.Cq, 11.10.Gh, 11.27.+d

keywords: Weyl frames; conformal transformations; general relativity.

address: Departamento de Física, Universidade Federal da Paraíba, João Pessoa, PB 58059-970, Brazil

\section{Introduction}

We would like to start by raising two questions of a very general character. The first questions is: What kind of invariance should the basic laws of physics possess? It is perhaps pertinent here to quote the following words by Dirac: "It appears as one of the fundamental principles of nature that the equations expressing the basic laws of physics should be invariant under the widest possible group of transformations" [1]. The second

*cromero@fisica.ufpb.br 
question, which seems to be of a rather epistemological character, is: To what extent is Riemannian geometry the only possible geometrical setting for general relativity? The purpose of the present work is to address, at least partially, these two questions.

It is a very well known fact that the principle of general covariance has played a major role in leading Einstein to the formulation of the theory of general relativity [2]. The idea underlying this principle is that coordinate systems are merely mathematical constructions to conveniently describe physical phenomena, and hence should not be an essential part of the fundamental laws of physics. In a more precise mathematical language, what is being required is that the equations of physics be expressed in terms of intrinsic geometrical objects, such as scalars, tensors or spinors, defined in the spacetime manifold. This mathematical requirement is sufficient to garantee the invariance of the form of the physical laws (or covariance of the equations) under arbitrary coordinate transformations. In field theories, one way of constructing covariant equations is to start with an action in which the Lagrangian density is a scalar function of the fields. In the case of general relativity, as we know, the covariance of the Einstein equations is a direct consequence of the invariance of the Einstein-Hilbert action with respect to space-time diffeomorphisms.

A rather different kind of invariance that has been considered in some branches of physics is invariance under conformal transformations. These represent changes in the units of length and time that differ from point to point in the space-time manifold. Conformal transformations were first introduced in physics by $\mathrm{H}$. Weyl in his attempt to formulate a unified theory of gravitation and electromagnetism [3]. However, in order to introduce new degrees of freedom to account for the electromagnetic field Weyl had to assume that the space-time manifold is not Riemannian. This extension consists of introducing an extra geometrical entity in the space-time manifold, a 1-form field $\sigma$, in terms of which the Riemannian compatibility condition between the metric $g$ and the connection $\nabla$ is redefined. Then, a group of transformations, which involves both $g$ and $\sigma$, is defined by requiring that under these transformations the new compatibility condition remain invariant. In a certain sense, this new invariance group, which we shall call the group of Weyl transformations, includes the conformal transformations as subgroup.

It turns out that Einstein's theory of gravity in its original formulation is not invariant neither under conformal transformations nor under Weyl transformations. One reason for this is that the geometrical language of Einstein's theory is completely based on Riemannian geometry. Indeed, for a long time general relativity has been inextricably associated with the geometry of Riemann. Further developments, however, have led to the discovery of different geometrical structures, which we might generically call "non-Riemannian" geometries, Weyl geometry being one of the first examples. Many of these developments were closely related to attempts at unifying gravity and electromagnetism [4]. While the newborn non-Riemannian geometries were invariably associated with new gravity theories, one question that naturally arises is to what extent is Riemannian geometry the only possible geometrical setting for the formulation of general relativity. One of our aims in this paper is to show that, surprisingly enough, one can formulate general relativity using the language of a non-Riemannian geometry, namely, the one known as Weyl integrable geometry. In this formulation, general relativity appears as a theory in which the gravitational field is described simultaneously by two geometrical fields: the metric tensor and the Weyl scalar field, the latter being an essential part of the geometry, manifesting its presence in almost all geometrical phenomena, such as curvature, geodesic motion, and 
so on. As we shall see, in this new geometrical setting general relativity exhibits a new kind of invariance, namely, the invariance under Weyl transformations.

The outline of this paper is as follows. We begin by presenting the basic mathematical facts of Weyl geometry and the concept of Weyl frames. In section 3, we show how to recast general relativity in the language of Weyl integrable geometry. In this formulation, we shall see that the theory is manifestly invariant under the group of Weyl transformations. We proceed, in section 4, to obtain the field equations and interpret the new form of the theory as a kind of scalar-tensor theory of gravity. In sections 5 and 6 , we explore the similarities of the formalism with Brans-Dicke theory of gravity. We devote section 7 to examine the Newtonian limit to get some insight into the meaning of the scalar field in the Weyl representation of general relativity. Then, in section 8 , we briefly illustrate how different pictures of the same phenomena may arise in distinct frames. In section 9, we show that the so-called WIST gravity theories are mathematically equivalent to Brans-Dicke theory when viewed in a particular frame, the Riemann frame. We end up with some remarks in section 10.

\section{Weyl Geometry}

The geometry conceived by Weyl is a simple generalization of Riemannian geometry. Instead of postulating that the covariant derivative of the metric tensor $g$ is zero, we assume the more general condition [3]

$$
\nabla_{\alpha} g_{\mu \nu}=\sigma_{\alpha} g_{\mu \nu}
$$

where $\sigma_{\alpha}$ denotes the components with respect to a local coordinate basis $\left\{\frac{\partial}{\partial x^{\alpha}}\right\}$ of a one-form field $\sigma$ defined on the manifold $M$. This represents a generalization of the Riemannian condition of compatibility between the connection $\nabla$ and $g$, namely, the requirement that the length of a vector remain unaltered by parallel transport [5]. If $\sigma$ vanishes, then (1) reduces to the familiar Riemannian metricity condition. It is interesting to note that the Weyl condition (1) remains unchanged when we perform the following simultaneous transformations in $g$ and $\phi$ :

$$
\begin{gathered}
\bar{g}=e^{f} g, \\
\bar{\sigma}=\sigma+d f,
\end{gathered}
$$

where $f$ is a scalar function defined on $M$. If $\sigma=d \phi$, where $\phi$ is a scalar field, then we have what is called a Weyl integrable manifold. The set $(M, g, \phi)$ consisting of a differentiable manifold $M$ endowed with a metric $g$ and a Weyl scalar field $\phi$ will be referred to as a Weyl frame. In the particular case of a Weyl integrable manifold (3) becomes

$$
\bar{\phi}=\phi+f .
$$

It turns out that if the Weyl connection $\nabla$ is assumed to be torsionless, then by virtue of condition (1) it gets completely determined by $g$ and $\sigma$. Indeed, a straightforward calculation shows that the components of the affine connection with respect to an arbitrary vector basis completely are given by

$$
\Gamma_{\mu \nu}^{\alpha}=\left\{\begin{array}{l}
\alpha \\
\mu \nu
\end{array}\right\}-\frac{1}{2} g^{\alpha \beta}\left[g_{\beta \mu} \sigma_{\nu}+g_{\beta \nu} \sigma_{\mu}-g_{\mu \nu} \sigma_{\beta}\right],
$$


where $\left\{\begin{array}{c}\alpha \\ \mu \nu\end{array}\right\}=\frac{1}{2} g^{\alpha \beta}\left[g_{\beta \mu, \nu}+g_{\beta \nu, \mu}-g_{\mu \nu,}\right]$ represents the Christoffel symbols, i.e., the components of the Levi-Civita connection 1 . An important fact that deserves to be mentioned is the invariance of the affine connection coefficients $\Gamma_{\mu \nu}^{\alpha}$ under the Weyl transformations (2) and (3). If $\sigma=d \phi$, (15) becomes

$$
\Gamma_{\mu \nu}^{\alpha}=\left\{\begin{array}{l}
\alpha \\
\mu \nu
\end{array}\right\}-\frac{1}{2} g^{\alpha \beta}\left[g_{\beta \mu} \phi,{ }_{\nu}+g_{\beta \nu} \phi, \mu-g_{\mu \nu} \phi, \beta\right] .
$$

A clear geometrical insight on the properties of Weyl parallel transport is given by the following proposition: Let $M$ be a differentiable manifold with an affine connection $\nabla$, a metric $g$ and a Weyl field of one-forms $\sigma$. If $\nabla$ is compatible with $g$ in the Weyl sense, i.e. if (11) holds, then for any smooth curve $C=C(\lambda)$ and any pair of two parallel vector fields $V$ and $U$ along $C$, we have

$$
\frac{d}{d \lambda} g(V, U)=\sigma\left(\frac{d}{d \lambda}\right) g(V, U)
$$

where $\frac{d}{d \lambda}$ denotes the vector tangent to $C$ and $\sigma\left(\frac{d}{d \lambda}\right)$ indicates the aplication of the 1-form $\sigma$ on $\frac{d}{d \lambda}$. (In a coordinate basis, putting $\frac{d}{d \lambda}=\frac{d x^{\alpha}}{d \lambda} \frac{\partial}{\partial x^{\alpha}}, V=V^{\beta} \frac{\partial}{\partial x^{\beta}}, U=U^{\mu} \frac{\partial}{\partial x^{\mu}}, \sigma=\sigma_{\nu} d x^{\nu}$, the above equation reads $\frac{d}{d \lambda}\left(g_{\alpha \beta} V^{\alpha} U^{\beta}\right)=\sigma_{\nu} \frac{d x^{\nu}}{d \lambda} g_{\alpha \beta} V^{\alpha} U^{\beta}$.)

If we integrate the equation (17) along the curve $C$ from a point $P_{0}=C\left(\lambda_{0}\right)$ to an arbitrary point $P=C(\lambda)$, then we obtain

$$
g(V(\lambda), U(\lambda))=g\left(V\left(\lambda_{0}\right), U\left(\lambda_{0}\right)\right) e^{\int_{\lambda_{0}}^{\lambda} \sigma\left(\frac{d}{d \rho}\right) d \rho} .
$$

If we put $U=V$ and denote by $L(\lambda)$ the length of the vector $V(\lambda)$ at $P=C(\lambda)$, then it is easy to see that in a local coordinate system $\left\{x^{\alpha}\right\}$ the equation (17) reduces to

$$
\frac{d L}{d \lambda}=\frac{\sigma_{\alpha}}{2} \frac{d x^{\alpha}}{d \lambda} L
$$

Consider the set of all closed curves $C:[a, b] \in R \rightarrow M$, i.e, with $C(a)=C(b)$. Then, we have the equation

$$
g(V(b), U(b))=g(V(a), U(a)) e e^{\oint\left(\frac{d}{d \lambda}\right) d \lambda} .
$$

It follows from Stokes' theorem that if $\sigma$ is an exact form, that is, if there exists a scalar function $\phi$, such that $\sigma=d \phi$, then

$$
\oint \sigma\left(\frac{d}{d \lambda}\right) d \lambda=0
$$

for any loop. In this case the integral $e^{\int_{\lambda_{0}}^{\lambda} \sigma\left(\frac{d}{d \rho}\right) d \rho}$ does not depend on the path and (8) may be rewritten in the form

$$
e^{-\phi(x(\lambda))} g(V(\lambda), U(\lambda))=e^{-\phi\left(x\left(\lambda_{0}\right)\right)} g\left(V\left(\lambda_{0}\right), U\left(\lambda_{0}\right)\right) .
$$

This equation means that we have an isometry between the tangent spaces of the manifold at the points $P_{0}=C\left(\lambda_{0}\right)$ and $P=C(\lambda)$ in the "effective" metric $\widehat{g}=e^{-\phi} g$.

\footnotetext{
${ }^{1}$ Throughout this paper our convention is that Greek indices take values from 0 to $n-1$, where $n$ is the dimension of $M$.
} 
Let us have a closer look at the correspondence between the Riemannian and Weyl integrable geometries suggested by Eq. (9). The first point to note is that, because $\sigma=d \phi$ for some scalar field $\phi$, then if we define an "effective" metric $\widehat{g}=e^{-\phi} g$, the Weyl condition of compatibility (or, as it is sometimes called, the non-metricity condition), expressed by Eq. (1) or (7), is formally equivalent to the Riemannian condition imposed on $\widehat{g}$, namely,

$$
\nabla_{\alpha} \widehat{g}_{\mu \nu}=0
$$

It may be easily verified that (6) follows directly from $\nabla_{\alpha} \widehat{g}_{\mu \nu}=0$. This simple fact has interesting and useful consequences, and later will serve as a guidance in the formulation of general relativity in terms of Weyl integrable geometry. One consequence is that since $\widehat{g}=e^{-\phi} g$ is invariant under the Weyl transformations (2) and (4) any geometrical quantity constructed with and solely with $\widehat{g}$ is invariant. Clearly, these will also be invariant under the Weyl transformations (21) and (4). Thus, in addition to the connection coefficients $\widehat{\Gamma}_{\mu \nu}^{\alpha}=\Gamma_{\mu \nu}^{\alpha}$, other geometrical objects such as the components of the curvature tensor $\widehat{R}_{\mu \beta \nu}^{\alpha}=R_{\mu \beta \nu}^{\alpha}=\Gamma_{\beta \mu, \nu}^{\alpha}-\Gamma_{\mu \nu, \beta}^{\alpha}+\Gamma_{\rho \nu}^{\alpha} \Gamma_{\beta \mu}^{\rho}-\Gamma_{\rho \beta}^{\alpha} \Gamma_{\nu \mu}^{\rho}$, the components of the Ricci tensor $\widehat{R}_{\mu \nu}=R_{\mu \nu}=R_{\mu \alpha \nu}^{\alpha}$, the scalar curvature $\widehat{R}=\widehat{g}^{\mu \nu} \widehat{R}_{\mu \nu}=\widehat{g}^{\mu \nu} R_{\mu \nu}=e^{\phi} g^{\mu \nu} R_{\mu \nu}=e^{\phi} R$ are evidently invariant. Moreover, in a Weyl integrable manifold it would be more natural to require this kind of invariance to hold also in the definition of length, so we would redefine the arc length of a curve $x^{\mu}=x^{\mu}(\lambda)$ between $x^{\mu}(a)$ and $x^{\mu}(b)$ as

$$
\Delta s=\int_{a}^{b}\left(\widehat{g}_{\mu \nu} \frac{d x^{\mu}}{d \lambda} \frac{d x^{\nu}}{d \lambda}\right)^{\frac{1}{2}} d \lambda=\int_{a}^{b} e^{-\frac{\phi}{2}}\left(g_{\mu \nu} \frac{d x^{\mu}}{d \lambda} \frac{d x^{\nu}}{d \lambda}\right)^{\frac{1}{2}} d \lambda .
$$

A second point concerns the interplay between covariant and contravariant vectors in a Weyl integrable manifold. Let us examine how the isomorphism that exists between vectors and 1-forms is modified when the manifold is endowed with an additional geometric field $\phi$. This question seems to be relevant because, as we know, it is this duality that underlies the usual operations of raising and lowering indices of vectors and tensors. In a Weyl integrable manifold these operations make sense only if they fulfil the requirement of Weyl invariance. Thus, let us now briefly recall how we show, in the Riemannian context, that the tangent space $T_{p}(M)$ and the cotangent space $T_{p}^{*}(M)$ at a point $p \in M$ are isomorphic [6]. The key point is to define the mapping $\widetilde{V}: T_{p}(M) \rightarrow \mathbb{R}$ with $\widetilde{V}(U)=$ $g(U, V)$ for any $U \in T_{p}(M)$. It is not difficult to see that $\widetilde{V}$ is a 1 -form and that to any 1-form $\sigma \in T_{p}^{*}(M)$ there corresponds a unique vector $V \in T_{p}(M)$ such that $\sigma(U)=$ $g(U, V)$. Now, assuming that $\left\{e_{\mu}\right\}$ and $\left\{e^{\mu}\right\}$ constitute dual bases for $T_{p}(M)$ and $T_{p}^{*}(M)$, respectively, and putting $V=V^{\mu} e_{\mu}, \sigma=\sigma_{\nu} e^{\nu}$, we then have $\sigma_{\mu}=\sigma\left(e_{\mu}\right)=g\left(e_{\mu}, V\right)=$ $V^{\nu} g\left(e_{\nu}, e_{\mu}\right)$. In view of the fact that $\sigma$ and $V$ are isomorphic it is natural "to lower" the index $V^{\mu}$ by defining $V_{\mu} \equiv \sigma_{\mu}=g_{\nu \mu} V^{\nu}$, with $g_{\nu \mu} \equiv g\left(e_{\nu}, e_{\mu}\right)$. Of course this procedure is not invariant under Weyl transformations since the effective metric $\widehat{g}=e^{-\phi} g$ does not enter in any of the above operations. To remedy this situation it suffices to redefine the above algebra by replacing the Riemannian scalar product $g: T_{p}(M) \times T_{p}(M) \rightarrow$ $\mathbb{R}$ by a new scalar product given by the bilinear form $\widehat{g}: T_{p}(M) \times T_{p}(M) \rightarrow \mathbb{R}$ with $\widehat{g}(U, V)=e^{-\phi} g(U, V)$. In this way the operations of raising and lowering indices when carried out with $\widehat{g}$ are clearly invariant under (2) and (4).

Let us finally conclude this section with a few historical comments on Weyl gravitational theory. Weyl developed an entirely new geometrical framework to formulate 
his theory, the main goal of which was to unify gravity and electromagnetism. As is well known, although admirably ingenious, Weyl's gravitational theory turned out to be unacceptable as a physical theory, as was immediately realized by Einstein, who raised objections to the theory [5, 7]. Einstein's argument was that in a non-integrable Weyl geometry the existence of sharp spectral lines in the presence of an electromagnetic field would not be possible since atomic clocks would depend on their past history [5]. However, the variant of Weyl geometry known as Weyl integrable geometry does not suffer from the drawback pointed out by Einstein. Indeed, it is the integral $I(a, b)=\int_{a}^{b} \sigma\left(\frac{d}{d \lambda}\right) d \lambda$ that is responsible for the difference between the readings of two identical atomic clocks following different paths. Because in Weyl integrable geometry $I(a, b)$ is not path-dependent the theory has attracted the attention of many cosmologists in recent years as a viable geometrical framework for gravity theories [8, 9].

\section{General Relativity and a New Kind of Invariance}

We have seen in the previous section that the Weyl compatibility condition (1) is preserved when we go from a frame $(M, g, \phi)$ to another frame $(M, \bar{g}, \bar{\phi})$ through the transformations (2) and (4). This has the consequence that the components $\Gamma_{\mu \nu}^{\alpha}$ of the affine connection are invariant under Weyl transformations, which, in turn, implies the invariance of the affine geodesics. Now, as is well known, geodesics plays a fundamental role in general relativity (GR) as well as in any metric theory of gravity. Indeed, an elegant aspect of the geometrization of the gravitational field lies in the geodesics postulate, i.e. the statement that light rays and particles moving under the influence of gravity alone follow space-time geodesics. Therefore a great deal of information about the motion of particles in a given space-time is promptly available once one knows its geodesics. The fact that geodesics

are invariant under (2) and (41) and that Riemannian geometry is a particular case of Weyl geometry (when $\sigma$ vanishes, or $\phi$ is constant) seems to suggest that it should be possible to express general relativity in a more general geometrical setting, namely, one in which the form of the field equations is also invariant under Weyl transformations. In this section, we shall show that this is indeed possible, and we shall proceed through the following steps. First, we shall assume that the space-time manifold which represents the arena of physical phenomena may be described by a Weyl integrable geometry, which means that gravity will be described by two geometric entities: a metric and a scalar field. The second step is to set up an action $S$ invariant under Weyl transformations. We shall require that $S$ be chosen such that there exists a unique frame in which it reduces to the Einstein-Hilbert action. The third step consists of extending Einstein's geodesic postulate to arbitrary frames, such that in the Riemann frame it should describe the motion of test particles and light exactly in the same way as predicted by general relativity. Finally, the fourth step is to define proper time in an arbitrary frame. This definition should be invariant under Weyl transformations and coincide with the definition of GR's proper time in the Riemann frame. It turns out then that the simplest action that can be built under these conditions is

$$
S=\int d^{4} x \sqrt{-g} e^{-\phi}\left\{R+2 \Lambda e^{-\phi}+\kappa e^{-\phi} L_{m}\right\}
$$

where $R$ denotes the scalar curvature defined in terms of the Weyl connection, $\Lambda$ is the cosmological constant, $L_{m}$ stands for the Lagrangian of the matter fields and $\kappa$ is the 
Einstein's constant 2. In $n$-dimensions we would have

$$
S_{n}=\int d^{n} x \sqrt{-g} e^{\left(1-\frac{n}{2}\right) \phi}\left\{R+2 \Lambda e^{-\phi}+\kappa e^{-\phi} L_{m}\right\} .
$$

In order to see that the above action is, in fact, invariant with respect to Weyl transformations, we just need to recall that under (21) and (4) we have $\bar{g}^{\mu \nu}=e^{-f} g^{\mu \nu}$, $\sqrt{-\bar{g}}=e^{\frac{n}{2} f} \sqrt{-g}, \bar{R}_{\nu \alpha \beta}^{\mu}=R_{\nu \alpha \beta}^{\mu}, \bar{R}_{\mu \nu}=R_{\mu \nu}, \bar{R}=\bar{g}^{\alpha \beta} \bar{R}_{\alpha \beta}=e^{-f} g^{\alpha \beta} R_{\alpha \beta}=e^{-f} R$. It will be assumed that $L_{m}$ depends on $\phi, g_{\mu \nu}$ and the matter fields, here generically denoted by $\xi$, its form being obtained from the special theory of relativity through the prescription $\eta_{\mu \nu} \rightarrow e^{-\phi} g_{\mu \nu}$ and $\partial_{\mu} \rightarrow \nabla_{\mu}$, where $\nabla_{\mu}$ denotes the covariant derivative with respect to the Weyl affine connection. If we designate the Lagrangian of the matter fields in special relativity by $L_{m}^{s r}=L_{m}^{s r}(\eta, \xi, \partial \xi)$, then the form of $L_{m}$ will be given by the rule $L_{m}(g, \phi, \xi, \nabla \xi) \equiv L_{m}^{s r}\left(e^{-\phi} g, \xi, \nabla \xi\right)$. As it can be easily seen, these rules also ensure the invariance under Weyl transformations of part of the action that is responsible for the coupling of matter with the gravitational field, and, at the same time, reproduce the principle of minimal coupling adopted in general relativity when we set $\phi=0$, that is, when we go to the Riemann frame by a Weyl transformation.

We now turn our attention to the motion of test particles and light rays. Here, our task is to extend GR's geodesic postulate in such a way to make it invariant under Weyl transformations. The extension is straightforward and may be stated as follows: if we represent parametrically a timelike curve as $x^{\mu}=x^{\mu}(\lambda)$, then this curve will correspond to the world line of a particle free from all non-gravitational forces, passing through the events $x^{\mu}(a)$ and $x^{\mu}(b)$, if and only if it extremizes the functional

$$
\Delta \tau=\int_{a}^{b} e^{-\frac{\phi}{2}}\left(g_{\mu \nu} \frac{d x^{\mu}}{d \lambda} \frac{d x^{\nu}}{d \lambda}\right)^{\frac{1}{2}} d \lambda
$$

which is obtained from the special relativistic expression of proper time by using the prescription $\eta_{\mu \nu} \rightarrow e^{-\phi} g_{\mu \nu}$. Clearly, the right-hand side of this equation is invariant under Weyl transformations and reduces to the known expression of the propertime in general relativity in the Riemann frame. We take $\Delta \tau$, as given above, as the extension to an arbitrary Weyl frame, of GR's clock hypothesis, i.e. the assumption that $\Delta \tau$ measures the proper time measured by a clock attached to the particle [10].

It is not difficult to verify that the extremization condition of the functional (13) leads to the equations

$$
\frac{d^{2} x^{\mu}}{d \lambda^{2}}+\left(\left\{\begin{array}{c}
\mu \\
\alpha \beta
\end{array}\right\}-\frac{1}{2} g^{\mu \nu}\left(g_{\alpha \nu} \phi_{, \beta}+g_{\beta \nu} \phi_{, \alpha}-g_{\alpha \beta} \phi_{, \nu}\right)\right) \frac{d x^{\alpha}}{d \lambda} \frac{d x^{\beta}}{d \lambda}=0,
$$

where $\left\{\begin{array}{l}\mu \\ \alpha \beta\end{array}\right\}$ denotes the Christoffel symbols calculated with $g_{\mu \nu}$. Let us recall that in the derivation of the above equations the parameter $\lambda$ has been choosen such that

$$
e^{-\phi} g_{\alpha \beta} \frac{d x^{\alpha}}{d \lambda} \frac{d x^{\beta}}{d \lambda}=K=\text { const }
$$

\footnotetext{
${ }^{2}$ Throughout this paper we shall adopt the following convention in the definition of the Riemann and Ricci tensors: $R_{\mu \beta \nu}^{\alpha}=\Gamma_{\beta \mu, \nu}^{\alpha}-\Gamma_{\mu \nu, \beta}^{\alpha}+\Gamma_{\rho \nu}^{\alpha} \Gamma_{\beta \mu}^{\rho}-\Gamma_{\rho \beta}^{\alpha} \Gamma_{\nu \mu}^{\rho} ; R_{\mu \nu}=R_{\mu \alpha \nu}^{\alpha}$. In this convention, we shall write the Einstein equations as $R_{\mu \nu}-\frac{1}{2} R g_{\mu \nu}-\Lambda g_{\mu \nu}=-\kappa T_{\mu \nu}$, with $\kappa=\frac{8 \pi G}{c 4}$.
} 
along the curve, which, up to an affine transformation, permits the identification of $\lambda$ with the proper time $\tau$. It turns out that these equations are exactly those that yield the affine geodesics in a Weyl integrable space-time, since they can be rewritten as

$$
\frac{d^{2} x^{\mu}}{d \tau^{2}}+\Gamma_{\alpha \beta}^{\mu} \frac{d x^{\alpha}}{d \tau} \frac{d x^{\beta}}{d \tau}=0
$$

where $\Gamma_{\alpha \beta}^{\mu}=\left\{\begin{array}{l}\mu \\ \alpha \beta\end{array}\right\}-\frac{1}{2} g^{\mu \nu}\left(g_{\alpha \nu} \phi_{, \beta}+g_{\beta \nu} \phi_{, \alpha}-g_{\alpha \beta} \phi_{, \nu}\right)$, according to ([6]), may be identified with the components of the Weyl connection. Therefore, the extension of the geodesic postulate by requiring that the functional $(13)$ be an extremum is equivalent to postulating that the particle motion must follow affine geodesics defined by the Weyl connection $\Gamma_{\alpha \beta}^{\mu}$. It will be noted that, as a consequence of the Weyl compatibility condition (1) between the connection and the metric, (14) holds automatically along any affine geodesic determined by (15). Because both the connection components $\Gamma_{\alpha \beta}^{\mu}$ and the proper time $\tau$ are invariant when we switch from one Weyl frame to the other, the equations (15) are invariant under Weyl transformations.

As we know, the geodesic postulate not only makes a statement about the motion of particles, but also regulates the propagation of light rays in space-time. Because the path of light rays are null curves, one cannot use the proper time as a parameter to describe them. In fact, light rays are supposed to follow null affine geodesics, which cannot be defined in terms of the functional (13), but, instead, they must be characterized by their behaviour with respect to parallel transport. We shall extend this postulate by simply assuming that light rays follow Weyl null affine geodesics.

It is well known that null geodesics are preserved under conformal transformations, although one needs to reparametrize the curve in the new gauge. In the case of Weyl transformations, null geodesics are also invariant with no need of reparametrization, since, again, the connection components $\Gamma_{\alpha \beta}^{\mu}$ do not change under (2) and (44), while the condition (14) is obvioulsy not altered. As a consequence, the causal structure of space-time remains unchanged in all Weyl frames. This seems to complete our program of formulating general relativity in a geometrical setting that exhibits a new kind of invariance, namely, that with respect to Weyl transformations 3 .

\section{General Relativity as a Scalar-Tensor Theory}

In the present formalism it is interesting to rewrite the action (12) in Riemannian terms. This is done by expressing the Weyl scalar curvature $R$ in terms of the Riemannian scalar curvature $\widetilde{R}$ and the scalar field $\phi$, which gives

$$
R=\widetilde{R}-(n-1) \square \phi+\frac{(n-1)(n-2)}{4} g^{\mu \nu} \phi_{, \mu} \phi_{, \nu},
$$

where $\square \phi$ denotes the Laplace-Beltrami operator. It is easily shown that, by inserting $R$ as given by (16) into Eq. (12) and using Gauss' theorem to neglect divergence terms in the integral, one obtains

$$
S_{n}=\int d^{n} x \sqrt{-g} e^{\left(1-\frac{n}{2}\right) \phi}\left\{\widetilde{R}+\omega g^{\mu \nu} \phi_{, \mu} \phi_{, \nu}+2 \Lambda e^{-\phi}+\kappa e^{-\phi} L_{m}\right\},
$$

\footnotetext{
${ }^{3}$ We found that, in [11, a similar action, in the case of vacuum, was obtained by using an argument based on the Palatini approach.
} 
where $\omega=\frac{(n-1)(2-n)}{4}$. For $n=4$ we have $\omega=-\frac{3}{2}$ and the action becomes

$$
S=\int d^{4} x \sqrt{-g} e^{-\phi}\left\{\widetilde{R}-\frac{3}{2} g^{\mu \nu} \phi_{, \mu} \phi_{, \nu}+2 \Lambda e^{-\phi}+\kappa e^{-\phi} L_{m}\right\} .
$$

In the next section, it will be convenient to change the scalar field variable $\phi$ by defining $\Phi=e^{-\phi}$. In terms of the new field $\Phi$, the action (18) takes the form

$$
S=\int d^{4} x \sqrt{-g}\left\{\Phi \widetilde{R}-\frac{3}{2 \Phi} g^{\mu \nu} \Phi_{, \mu} \Phi_{, \nu}+2 \Lambda \Phi^{2}+\kappa \Phi^{2} L_{m}\right\}
$$

If we take variations of $S$, as given by (18), with respect to $g_{\mu \nu}$ and $\phi$, these being considered as independent fields, we shall obtain, respectively,

$$
\begin{gathered}
\widetilde{G}_{\mu \nu}-\phi_{, \mu ; \nu}+g_{\mu \nu} \square \phi-\frac{1}{2}\left(\phi_{, \mu} \phi_{, \nu}+\frac{1}{2} g_{\mu \nu} \phi_{, \alpha} \phi^{, \alpha}\right)=e^{-\phi} \Lambda g_{\mu \nu}-\kappa T_{\mu \nu}, \\
\widetilde{R}-3 \square \phi+\frac{3}{2} \phi_{, \alpha} \phi^{, \alpha}=\kappa T-4 e^{-\phi} \Lambda
\end{gathered}
$$

where $\widetilde{G}_{\mu \nu}$ and $\widetilde{R}$ denotes the Einstein tensor and the curvature scalar, both calculated with the Riemannian connection, and $T=g^{\mu \nu} T_{\mu \nu}$. It should be noted that (21) is just the trace of (20), and so, the above equations are not independent. This is consistent with the fact that we have complete freedom in the choice of the Weyl frame. It also means that $\phi$ may be viewed as an arbitrary gauge function and not as a dynamical field.

It is straightforward to verify that in terms of the variable $\Phi=e^{-\phi}$, the equations (20) and (21) read

$$
\begin{gathered}
\widetilde{G}_{\mu \nu}=-\kappa T_{\mu \nu}+\Lambda \Phi g_{\mu \nu}+\frac{3}{2 \Phi^{2}}\left(\Phi_{, \mu} \Phi_{, \nu}-\frac{1}{2} g_{\mu \nu} \Phi_{, \alpha} \Phi^{, \alpha}\right)-\frac{1}{\Phi}\left(\Phi_{, \mu ; \nu}-g_{\mu \nu} \square \Phi\right), \\
\widetilde{R}+3 \frac{\square \Phi}{\Phi}-\frac{3}{2 \Phi^{2}} \Phi_{, \alpha} \Phi^{, \alpha}=\kappa T-4 \Phi \Lambda .
\end{gathered}
$$

Some considerations should be made on the form taken by the energy-momentum tensor $T_{\mu \nu}$, which appears on the right-hand side of the equations (20) and (22). Here, as well as in the previous development of the formalism that leads to the formulation of general relativity in a Weyl integrable manifold, we use the effective metric $\widehat{g}=e^{-\phi} g$ as a guide to ensure Weyl invariance. In this way, it is natural to define the energy-momentum tensor $T_{\mu \nu}(\phi, g, \xi, \nabla \xi)$ of the matter field $\xi$, in an arbitrary Weyl frame $(M, g, \phi)$, by the formula

$$
\delta \int d^{4} x \sqrt{-g} e^{-2 \phi} L_{m}(g, \phi, \xi, \nabla \xi)=\int d^{4} x \sqrt{-g} e^{-2 \phi} T_{\mu \nu}(\phi, g, \xi, \nabla \xi) \delta\left(e^{\phi} g^{\mu \nu}\right),
$$

where the variation on the left-hand side must be carried out simultaneously with respect to both $g_{\mu \nu}$ and $\phi$. In order to see that the above definition makes sense, first recall that $L_{m}(g, \phi, \xi, \nabla \xi)$ is given by the prescription $\eta_{\mu \nu} \rightarrow e^{-\phi} g_{\mu \nu}$ and $\partial_{\mu} \rightarrow \nabla_{\mu}$, where $\nabla_{\mu}$ denotes the covariant derivative with respect to the Weyl affine connection. Let us recall here that $L_{m}(g, \phi, \xi, \nabla \xi) \equiv L_{m}^{s r}\left(e^{-\phi} g, \xi, \nabla \xi\right)$, where $L_{m}^{s r}$ denotes the Lagrangian of the field $\xi$ in flat Minkowski space-time. Secondly, it should be clear that the left-hand side of the equation 
(24) can always be put in the same form of the right-hand side of the same equation. This can easily be seen from the fact that $\delta L_{m}=\frac{\partial L_{m}}{\partial g^{\mu \nu}} \delta g^{\mu \nu}+\frac{\partial L_{m}}{\partial \phi} \delta \phi=\frac{\partial L_{m}}{\partial\left(e^{\phi} g^{\mu \nu}\right)} \delta\left(e^{\phi} g^{\mu \nu}\right)$ and that $\delta\left(\sqrt{-g} e^{-2 \phi}\right)=-\frac{1}{2} \sqrt{-g} e^{-3 \phi} g_{\mu \nu} \delta\left(e^{\phi} g^{\mu \nu}\right)$. Finally, it is clear that the definition of $T_{\mu \nu}(\phi, g, \xi, \nabla \xi)$ given by (24) is invariant under the Weyl transformations (2) and (44).

We would like to conclude this section with a brief comment on the form that the equation that expresses the energy-momentum conservation law takes in a arbitrary Weyl frame. We start with the Einstein's equations written in the Riemann frame $(M, \widehat{g}, 0)$ :

$$
G_{\mu \nu}(\widehat{g}, 0)=-\kappa T_{\mu \nu}(\widehat{g}, 0) .
$$

Because $G_{\mu \nu}(\widehat{g}, 0)$ is divergenceless with respect to the metric connection $\left\{\begin{array}{l}\alpha \\ \mu \nu\end{array}\right\}_{\widehat{g}}=\frac{1}{2} \widehat{g}^{\alpha \beta}\left[\widehat{g}_{\beta \mu, \nu}+\right.$ $\left.\widehat{g}_{\beta \nu, \mu}-\widehat{g}_{\mu \nu, \beta}\right]$ it follows from (25) that

$$
\widehat{\nabla}_{\alpha} T_{\mu}^{\alpha}=\widehat{\nabla}_{\alpha}\left(\widehat{g}^{\alpha \nu} T_{\mu \nu}\right)=0,
$$

where the symbol $\widehat{\nabla}_{\alpha}$ denotes the covariant derivative defined by $\left\{\begin{array}{c}\alpha \\ \mu \nu\end{array}\right\}_{\widehat{g}}$. If we now go to an arbitrary Weyl frame $\left(M, g=e^{\phi} \widehat{g}, \phi\right)$, then a straightforward calculation shows that (26) takes the form

$$
\nabla_{\alpha} T_{\mu}^{\alpha}=T_{\mu}^{\alpha} \phi_{, \alpha}-\frac{1}{2} T \phi_{, \mu}
$$

where $T=g^{\alpha \beta} T_{\alpha \beta}$ and $\nabla_{\alpha}$ stands for the covariant derivative defined by the metric connection calculated with $g$.

At first sight, due to the presence of non-vanishing terms on the right-hand side of (27) one may be led to think that in the Weyl frame we have an apparent violation of the energy-momentum conservation law. Nonetheless, we must remember that if one is not working in the Riemann frame the Weyl scalar field $\phi$ is an essential part of the geometry and necessarily should appear in any equation describing the behaviour of matter in space-time. This explain the presence of $\phi$ coupled with $T_{\mu \nu}$ in (27). Note that if $\phi=$ const we recover the familiar general-relativistic energy-momentum conservation equation. Finally, it is not difficult to verify that the above equation is invariant under the Weyl transformations (2) and (4).

\section{Similarities with Brans-Dicke theory}

We shall now take a look at some similarities between the Brans-Dicke theory of gravity and general relativity, when the latter is expressed in the formalism we have developed in the previous section. For this purpose, let us recall that the field equations of Brans-Dicke theory of gravity may be written in the form [12]

$$
\begin{gathered}
\widetilde{G}_{\mu \nu}=-\frac{\kappa^{*}}{\Phi} T_{\mu \nu}-\frac{\omega}{\Phi^{2}}\left(\Phi_{, \mu} \Phi_{, \nu}-\frac{1}{2} g_{\mu \nu} \Phi_{, \alpha} \Phi^{, \alpha}\right)-\frac{1}{\Phi}\left(\Phi_{, \mu ; \nu}-g_{\mu \nu} \square \Phi\right), \\
\widetilde{R}-2 \omega \frac{\square \Phi}{\Phi}+\frac{\omega}{\Phi^{2}} \Phi_{, \alpha} \Phi^{, \alpha}=0,
\end{gathered}
$$

where $\kappa^{*}=\frac{8 \pi}{c^{4}}$, and we are keeping the notation of the previous section, in which $\widetilde{G}_{\mu \nu}$ and $\widetilde{R}$ denotes the Einstein tensor and the curvature scalar calculated with respect to the metric $g_{\mu \nu}$. By combining (28) and (29) we can easily derive the equation

$$
\square \Phi=\frac{\kappa^{*} T}{2 \omega+3},
$$


which is the most common form of the scalar field equation usually found in the literature. The equation (30), however, is not defined for $\omega=-\frac{3}{2}$, so for this value of $\omega$ one has to use (29) instead, which then, becomes

$$
\widetilde{R}+3 \frac{\square \Phi}{\Phi}-\frac{3}{2 \Phi^{2}} \Phi_{, \alpha} \Phi^{, \alpha}=0 .
$$

On the other hand, the equation (28) for $\omega=-\frac{3}{2}$ reads

$$
\widetilde{G}_{\mu \nu}=-\frac{\kappa^{*}}{\Phi} T_{\mu \nu}+\frac{3}{2 \Phi^{2}}\left(\Phi_{, \mu} \Phi_{, \nu}-\frac{1}{2} g_{\mu \nu} \Phi_{, \alpha} \Phi^{, \alpha}\right)-\frac{1}{\Phi}\left(\Phi_{, \mu ; \nu}-g_{\mu \nu} \square \Phi\right) .
$$

Now, if we take the trace of the (32) with respect to $g_{\mu \nu}$ we get

$$
\widetilde{R}+3 \frac{\square \Phi}{\Phi}-\frac{3}{2 \Phi^{2}} \Phi_{, \alpha} \Phi^{, \alpha}=\frac{\kappa^{*}}{\Phi} T \text {. }
$$

Of course (31) and (33) are not compatible, unless $T=0$, which, then, implies that when $\omega=-\frac{3}{2}$ the Brans-Dicke field equations (28) and (29) cease to be independent, and the system of differential equations for $g_{\mu \nu}$ and $\Phi$ becomes undertermined. As a consequence, one may freely choose an arbitrary $\Phi$ and work out a solution for $g_{\mu \nu}$ from (32). In particular, one can set $\Phi=\Phi_{0}=$ const, in which case (32) becomes formally identical to the Einstein equations constant with the gravitational constant $G$ replaced by $\frac{1}{\Phi_{0}}$. At this point, it is interesting to note that one gets the same result by means of the conformal transformation $\bar{g}_{\mu \nu}=e^{-\Phi} g_{\mu \nu}$, since the conformally transformed Einstein tensor $\bar{G}_{\mu \nu}$ is given by $\bar{G}_{\mu \nu}=\widetilde{G}_{\mu \nu}-\frac{3}{2 \Phi^{2}}\left(\Phi_{, \mu} \Phi_{, \nu}-\frac{1}{2} g_{\mu \nu} \Phi_{, \alpha} \Phi^{, \alpha}\right)+\frac{1}{\Phi}\left(\Phi_{, \mu ; \nu}-g_{\mu \nu} \square \Phi\right)$. ( It is curious that one could use this property to generate an infinite class of Brans-Dicke theory for $w=-\frac{3}{2}$ from known solutions of the Einstein equations.) This known mathematical fact is often interpreted in the literature as representing a conformal equivalence between Brans-Dicke gravity for $w=-3 / 2$ and general relativity [14, 15]. It will be noted, however, that, in spite of the amazing similarity of the field equations, we are far from having a complete analogy between the two theories. Indeed, when we turn to the motion of test particles, we immediately realize that in the Brans-Dicke theory it is postulated that these particles must follow Riemannian geodesics, whereas in the case of GR formulated in a Weyl frame (or in the case of conformal relativity) these must follow geodesics that are not Riemannian. In the next section, we shall illustrate this point with a simple example taken from a known vacuum solution of Brans-Dicke theory, namely, the O`Hanlon-Tupper vacuum solution [16].

\section{Brans-Dicke vacuum solutions for $\mathrm{w}=-3 / 2$}

In the case of vacuum and vanishing cosmological constant, the equations (22) and (23) reduce to

$$
\begin{gathered}
\widetilde{G}_{\mu \nu}=\frac{3}{2 \Phi^{2}}\left(\Phi_{, \mu} \Phi_{, \nu}-\frac{1}{2} g_{\mu \nu} \Phi_{, \alpha} \Phi^{, \alpha}\right)-\frac{1}{\Phi}\left(\Phi_{, \mu ; \nu}-g_{\mu \nu} \square \Phi\right), \\
\widetilde{R}+3 \frac{\square \Phi}{\Phi}-\frac{3}{2 \Phi^{2}} \Phi_{, \alpha} \Phi^{, \alpha}=0,
\end{gathered}
$$

respectively. As we have just mentioned, in this situation the equations of general relativity in an arbitrary Weyl frame $((\underline{22})$ and (23) $)$ are identical to those of Brans-Dicke 
theory $((28)$ and $(29))$ for $\omega=-\frac{3}{2}$, provided that we identify the Weyl scalar field with the Brans-Dicke scalar field. At this point, suppose we want to see how a solution of the above equations, regarded as a vacuum solution of Brans-Dicke theory for $\omega=-\frac{3}{2}$, would look like when interpreted as a vacuum solution of general relativity in a certain Weyl frame, where the Brans-Dicke scalar field $\Phi$ now plays the role of the Weyl scalar field. We can take, for instance, the well known $\mathrm{O}^{\prime}$ Hanlon-Tupper model, which is a vacuum solution of Brans-Dicke field equations corresponding to a homogeneous isotropic space-time with spatial flat section $(k=0)$. In this model, the metric $g_{\mu \nu}$ and the scalar field $\Phi$ are given, respectively, by

$$
d s^{2}=d t^{2}-A(t)^{2}\left(d r^{2}+r^{2} d \theta^{2}+r^{2} \sin ^{2} \theta d \varphi^{2}\right),
$$

where $A(t)=A_{0} t^{p}, \Phi=\Phi_{0} t^{q}$, with $p=\frac{1}{3 \omega+4}(\omega+1 \pm \sqrt{(2 \omega+3) / 3})$, and $q=\frac{1}{3 \omega+4}(1 \mp$ $\sqrt{3(2 \omega+3)}, A_{0}$ and $\Phi_{0}$ being integration constants [16]. For $w>-\frac{3}{2}$ this solution has a big bang singularity as $t \rightarrow 0$. When $\omega \rightarrow \infty$ it has the limit $A(t)=A_{0} t^{\frac{1}{3}}$, $\Phi(t)=\Phi_{0}=$ const, which is identical to the Friedmann model for stiff matter equation of state [17], and so this solution does not go over the corresponding general relativistic solution, i.e., Minkowski space-time [18, 19]. For $\omega=-\frac{3}{2}$ we have $A(t)=A_{0} t$ and $\Phi=\Phi_{0} t^{-2}$. This represents a model in which the so-called Dirac's hypothesis does not hold, since the Newtonian gravitational "constant", interpreted in Brans-Dicke theory as the inverse of the scalar field $(G \propto 1 / \Phi)$, decreases as the universe expands [20].

In order to interpret the $\mathrm{O}^{\prime}$ Hanlon-Tupper model in the light of a general relativistic picture, we start by putting (35a) in the conformally-flat form

$$
d s^{2}=e^{\Psi(\tau)}\left(d \tau^{2}-d r^{2}+r^{2} d \theta^{2}+r^{2} \sin ^{2} \theta d \varphi^{2}\right),
$$

where we have made the coordinate transformation $t=e^{A_{0} \tau}$ and defined $\Psi(\tau)=2(\tau+$ $\ln A_{0}$ ). In terms of the new coordinate, the Brans-Dicke scalar field is given by $\Phi=$ $\Phi_{0} A_{0}^{2} e^{-\Psi(\tau)}$. Regarding both $g_{\mu \nu}$ given by (36) and $\Phi$ as describing the gravitational field in the Weyl frame $(M, g, \Phi)$, we now want to know how they will appear in a Riemann frame $(M, \widehat{g}, \widehat{\Phi})$, that is, in a frame, where $\widehat{\Phi}$ is constant and, hence, the geometry is Riemannian. Recalling that the general form of theWeyl transformations (2) and (44) in terms of the variables $\Phi=e^{-\phi}$ and $\bar{\Phi}=e^{-\bar{\phi}}$ is given by

$$
\begin{gathered}
\widehat{g}_{\mu \nu}=e^{f} g_{\mu \nu}, \\
\widehat{\Phi}=e^{-f} \Phi,
\end{gathered}
$$

it is clear that the natural choice of $f$ that will turn $\Phi$ into a constant is $f=-\Psi(\tau)$. We thus are led to the Riemann frame $\left(M, \widehat{g}=\eta, \widehat{\Phi}=\Phi_{0} A_{0}^{2}\right)$, where $\eta$ denotes Minkowski metric. Therefore, we conclude that the $\mathrm{O}^{\prime}$ Hanlon-Tupper cosmological model, when regarded formally as a general relativistic solution in the Weyl frame $(M, g, \Phi)$, is equivalent to Minkowski space-time, whose geodesics consists of straight lines satisfying the equations

$$
\frac{d^{2} x^{\mu}}{d \tau^{2}}=0
$$

From the fact the affine geodesics are invariant under the Weyl transformations (2) and (4), and since in the Riemann frame $\left(M, \widehat{g}=\eta, \widehat{\Phi}=\Phi_{0} A_{0}^{2}\right)$ the Weyl affine geodesics

\footnotetext{
${ }^{4} \mathrm{O}^{\prime}$ Hanlon-Tupper solution for $\omega=-\frac{3}{2}$ is identical to the cosmological model found by Singh and Shridhar for a radiation-filled Roberton-Walker universe [21].
} 
coincide with the metric geodesics, it is evident that in the in the Weyl frame $(M, g, \Phi)$ the affine geodesics will also be given by (39).

As we have already pointed out, the formal equivalence exhibited above between BransDicke vacuum solutions for $w=-\frac{3}{2}$ and general relativistic vacuum solutions expressed in an Weyl geometric setting is not complete. The reason is that we have not taken into account an aspect that is fundamental to any metric theory of gravity: how do we determine the motion of test particles and light. Indeed, as we have mentioned earlier, in the case of general relativity, the geodesic equations that governs the motion of test particles and light in an arbitrary Weyl frame are constructed with the affine connection coefficients, which explicitly involves the Weyl scalar field, and are invariant under Weyl transformations. Of course we have a different situation in the case of Brans-Dicke theory, where, even in the presence of the scalar field, the geodesics are defined by the Levi-Civita connection. Therefore, in the $\mathrm{O}^{\prime}$ Hanlon-Tupper model the geodesic motion of particles and light will not be given by (39). A short calculation shows that the Brans-Dicke geodesic equations are

$$
\frac{d^{2} x^{\mu}}{d \tau^{2}}+\frac{d \Psi}{d \tau} \frac{d x^{\mu}}{d \tau}+\frac{e^{-\Psi}}{2} \Psi^{, \mu}=0
$$

To conclude this section, we would like to show how the formal equivalence discussed above can be used to generate a whole class of vacuum solutions of Brans-Dicke field equations for $\omega=-\frac{3}{2}$, which includes the $\mathrm{O}^{\prime}$ Hanlon-Tupper model as a particular case. To do this, let us suppose that we want to obtain a solution of the field equations (34) corresponding to a homogeneous and isotropic spacetime. As we know, the most general form of the metric of such spacetime may be written as

$$
d s^{2}=d t^{2}-\frac{A(t)^{2}}{1+\frac{k r^{2}}{4}}\left(d r^{2}+r^{2} d \theta^{2}+r^{2} \sin ^{2} \theta d \varphi^{2}\right),
$$

where $k=0, \pm 1$ represents the curvature of the spatial sections. We now regard (34) as the Einstein's field equations in the Weyl frame $(M, g, \Phi)$, so that we can go to the Riemann frame $(M, \bar{g}, \bar{\Phi}=1)$ through the Weyl transformations (37) and (38) by choosing $f=\ln \Phi$. In the Riemann frame, the line element corresponding to $\bar{g}$ will be

$$
d s^{2}=\Phi(t) d t^{2}-\frac{\Phi(t) A(t)^{2}}{1+\frac{k r^{2}}{4}}\left(d r^{2}+r^{2} d \theta^{2}+r^{2} \sin ^{2} \theta d \varphi^{2}\right)
$$

Defining a new time coordinate $\bar{t}$ by $\Phi(t)^{1 / 2} d t=d \bar{t}$ and putting $\Phi\left(t(\bar{t}) A^{2} t(\bar{t})=\bar{A}^{2}(\bar{t})\right.$, (40) takes the form

$$
d s^{2}=d \bar{t}^{2}-\frac{\bar{A}(\bar{t})^{2}}{1+\frac{k r^{2}}{4}}\left(d r^{2}+r^{2} d \theta^{2}+r^{2} \sin ^{2} \theta d \varphi^{2}\right) .
$$

Now, in the Riemann frame (34) becomes simply

$$
\bar{G}_{\mu \nu}=0
$$

with $\bar{G}_{\mu \nu}$ calculated with the metric $\bar{g}$. It may be readily verified that this yields only one independent equation, namely,

$$
\left(\frac{d \bar{A}}{d \bar{t}}\right)^{2}=-k c^{2}
$$


An obvious conclusion that can be drawn from the above equation is that there are no solutions for $k=1$ (this has been pointed out in ([15]). If we take $k=0$, then $\bar{A}(\bar{t})=B$, where $B$ is an arbitrary constant. Thus, from the definition of $\bar{A}(\bar{t})$, we have $\Phi(t) A(t)^{2}=B$. This means that we have an infinite number of Brans-Dicke vacuum solutions for $\omega=-\frac{3}{2}$, O'Hanlon-Tupper model merely corresponding to the particular choice $A(t) \sim t$.

\section{The Newtonian limit in a general Weyl frame}

In order to gain some insight into the meaning of this new representation of general relativity developed in the previous sections, let us now proceed to examine the Newtonian limit of general relativity in an arbitrary Weyl frame $(M, g, \phi)$.

As we know, a metric theory of gravity is said to possess a Newtonian limit in the non-relativistic weak-field regime if one can derive from it Newton's second law from the geodesic equations as well as the Poisson equation from the gravitational field equations. Let us see how general relatity when expressed in a form that is invariant under Weyl transformations fulfills these requirements. The method we shall employ here to treat this problem is standard and can be found in most textbooks on general relativity ( see, for instance, [13] ).

Since in Newtonian mechanics the space geometry is Euclidean, a weak gravitational field in a geometric theory of gravity should manifest itself as a metric phenomenon through a slight perturbation of the Minkowskian space-time metric. Thus we consider a time-independent metric tensor of the form

$$
g_{\mu \nu}=\eta_{\mu \nu}+\epsilon h_{\mu \nu}
$$

where $n_{\mu \nu}$ is the Minkowski tensor, $\epsilon$ is a small parameter and the term $\epsilon h_{\mu \nu}$ represents a very small time-independent perturbation due to the presence of some matter configuration. Because we are working in the non-relativistic regime we shall suppose that the velocity $V$ of the particle along the geodesic is much less then $c$, so that the paramenter

$\beta=\frac{V}{c}$ will be regarded as very small; hence in our calculations only first-order terms in $\epsilon$ and $\beta$ will be kept. The same kind of approximation will be assumed with respect to the Weyl scalar field $\phi$, which will be supposed to be static and small, i.e. of the same order as $\epsilon$, and to emphasise this fact we shall write $\phi=\epsilon \varphi$, where $\varphi$ is a finite function. Adopting then usual Minkowskian coordinates of special relativity we can write the line element defined by (44) as

$$
d s^{2}=\left(d x^{0}\right)^{2}-\left(d x^{1}\right)^{2}-\left(d x^{2}\right)^{2}-\left(d x^{3}\right)^{2}-\epsilon h_{\mu \nu} d x^{\mu} d x^{\nu},
$$

which leads, in our approximation, to

$$
\left(\frac{d s}{d t}\right)^{2} \cong c^{2}\left(1+\epsilon h_{00}\right)
$$

We shall now consider, in the same approximation, the geodesic equations

$$
\frac{d^{2} x^{\mu}}{d \tau^{2}}+\Gamma_{\alpha \beta}^{\mu} \frac{d x^{\alpha}}{d \tau} \frac{d x^{\beta}}{d \tau}=0
$$


recalling that the symbol $\Gamma_{\alpha \beta}^{\mu}$ designates the components of the Weyl affine connection. From (5) it is easy to verify that, to first order in $\epsilon$, we have

$$
\Gamma_{\mu \nu}^{\alpha}=\frac{\epsilon}{2} n^{\alpha \lambda}\left[h_{\lambda \mu, \nu}+h_{\lambda \nu, \mu}-h_{\mu \nu, \lambda}+n_{\mu \nu} \varphi_{, \lambda}-n_{\lambda \mu} \varphi_{, \nu}-n_{\lambda \nu} \varphi_{, \mu}\right]
$$

It is not difficult to see that, unless $\mu=\nu=0$, the product $\Gamma_{\alpha \beta}^{\mu} \frac{d x^{\alpha}}{d s} \frac{d x^{\beta}}{d s}$ is of order $\epsilon \beta$ or higher. In this way, the geodesic equations (46) become, to first order in $\epsilon$ and $\beta$

$$
\frac{d^{2} x^{\mu}}{d s^{2}}+\Gamma_{00}^{\mu}\left(\frac{d x^{0}}{d s}\right)^{2}=0 .
$$

By taking into account (45) the above equations may be written as

$$
\frac{d^{2} x^{\mu}}{d t^{2}}+c^{2} \Gamma_{00}^{\mu}=0
$$

Clearly for $\mu=0$ the equation (48) reduces to an identity. On the other hand, if $\mu$ is a spatial index, a simple calculation yields $\Gamma_{00}^{i}=-\frac{\epsilon}{2} \eta^{i j} \frac{\partial}{\partial x^{j}}\left(h_{00}-\varphi\right)$, hence the geodesic equation in this approximation becomes, in three-dimensional vector notation,

$$
\frac{d^{2} \vec{X}}{d t^{2}}=-\frac{\epsilon}{2} c^{2} \vec{\nabla}\left(h_{00}-\varphi\right),
$$

which is simply Newton's equation of motion in a classical gravitational field provided we identify the scalar gravitational potential with

$$
U=\frac{\epsilon c^{2}}{2}\left(h_{00}-\varphi\right) .
$$

It is worth noting the presence of the Weyl field $\varphi$ in the above equation. In fact, it is the combination $h_{00}-\varphi$ that represents the Newtonian potential.

Let us now turn our attention to the Newtonian limit of the field equations. For this purpose it will be convenient to recast the equation (20) with $\Lambda=0$ into the form

$$
R_{\mu \nu}=-\kappa T_{\mu \nu}+\frac{1}{2} g_{\mu \nu}\left(\kappa T+\square \phi-\phi, \alpha \phi^{, \alpha}\right)+\phi_{; \mu ; \nu}+\frac{1}{2} \phi_{, \mu} \phi_{, \nu} .
$$

In the weak-field approximation, i.e. when $g_{\mu \nu}=\eta_{\mu \nu}+\epsilon h_{\mu \nu}$, it is easy to show that to first order in $\epsilon$, we have $R_{00}=-\frac{1}{2} \nabla^{2} \epsilon h_{00}$, where $\nabla^{2}$ denotes the Laplacian operator in flat space-time. On the other hand, because we are assuming a static regime $\phi_{,_{0}}=0$, so the equation (50) for $\mu=\nu=0$ now reads

$$
\nabla^{2}\left[\frac{\epsilon c^{2}}{2}\left(h_{00}-\varphi\right)\right]=\kappa\left(T_{00}-T\right) .
$$

Let us consider a configuration of matter distribution with low proper density $\rho$ moving at non-relativistic speed. The energy-momentum tensor in this case is obtainable from special relativistic matter tensor

$$
T_{\mu \nu}=\left(\rho c^{2}+p\right) V_{\mu} V_{\nu}-p \eta_{\mu \nu}
$$


where $\rho, p$ and $V^{\mu}$ denotes, respectively, the proper density, pressure and velocity field. We now need the expression of $T_{\mu \nu}$ in an arbitrary Weyl frame. Rewriting this expression as $T_{\mu \nu}=\left(\rho c^{2}+p\right) \eta_{\mu \alpha} \eta_{\nu \gamma} V^{\alpha} V^{\gamma}-p \eta_{\mu \nu}$ and following the prescription $\eta_{\mu \nu} \rightarrow e^{-\phi} g_{\mu \nu}$, we obtain

$$
T_{\mu \nu}=\left(\rho c^{2}+p\right) e^{-2 \phi} g_{\mu \alpha} g_{\nu \gamma} V^{\alpha} V^{\gamma}-p e^{-\phi} g_{\mu \nu}
$$

which is the desired expression of the energy-momentum tensor in an arbitrary frame. It is worth noting that in going from (52) to (53) the quantities $\rho, p$ and $V^{\alpha}=\frac{d x^{\alpha}}{d \tau}$ are kept unaltered as, by definition, they are invariant under Weyl transformations. Putting $e^{-\phi} \simeq 1-\epsilon \varphi$ and recalling that in a non-relativistic regime we can neglect $p$ with respect to $\rho$, leads to $T_{00}=T \simeq \rho c^{2}$. In this way, we obtain, to first order in $\epsilon$

$$
T_{\mu \nu} \simeq \rho c^{2} \eta_{\mu \alpha} \eta_{\nu \gamma} V^{\alpha} V^{\gamma}
$$

Finally, after substituting $\kappa=\frac{8 \pi G}{c^{4}}$ into the Eq. (151) we obtain

$$
\nabla^{2}\left[\frac{\epsilon c^{2}}{2}\left(h_{00}-\varphi\right)\right]=4 \pi G \rho
$$

which clearly corresponds to the Poisson equation for the gravitational field $\nabla^{2} U=4 \pi G \rho$ with $U$ given by (49).

\section{Different pictures of the same physical phenomena}

As we have seen, when we go from one frame $(M, g, \phi)$ to another frame $(M, \bar{g}, \bar{\phi})$ through the Weyl transformations (2) and (4), the pattern of affine geodesic curves does not change. However, distinct geometrical and physical pictures may arise in different frames. This is particular evident in the case of a conformally flat space-time, i.e. when we have in a Riemann frame $g=e^{\phi} \eta$. In such situations, one can completely gauge away the Riemannian curvature by a frame transformation, thereby going to a frame in which one is left with a geometrical scalar field in a Minkowski background [22]. This is well illustrated, for instance, when we consider the class of Robertson-Walker (RW) spacetimes $(k=0, \pm 1)$, which are known to be conformally flat [23]. If we go to the Weyl frame $(M, \eta, \phi)$ by means of a Weyl transformation we arrive at a new cosmological scenario in which the Riemannian curvature ceases to determine the cosmic expansion and other phenomena, these effects being now attributed to the sole action of a scalar field living in flat space-time. There are many other examples of how distinct physical interpretations of the same phenomena are possible in different frames. By way of illustration, we shall consider, in this section, how one would describe, in a general Weyl frame, an important effect predicted by general relativity: the so-called gravitational spectral shift.

Let us consider the gravitational field generated by a massive body, which in an arbitrary Weyl frame $(M, g, \phi)$ is described by both the metric tensor $g_{\mu \nu}$ and the scalar field $\phi$. For the sake of simplicity, let us restrict ourselves to the case of a static field, in which neither $g_{\mu \nu}$ nor $\phi$ depends on time. Let us suppose that a light wave is emitted on the body at a fixed point with spatial coordinates $\left(r_{E}, \theta_{E}, \varphi_{E}\right)$ and received by an observer at fixed point $\left(r_{R}, \theta_{R}, \varphi_{R}\right)$. Denoting the coordinate times of emission and reception by $t_{E}$ and $t_{R}$, respectively, the light signal, which in the Weyl frame corresponds to a null affine geodesic, connects the event $\left(t_{E}, r_{E}, \theta_{E}, \varphi_{E}\right)$ with the event $\left(t_{R}, r_{R}, \theta_{R}, \varphi_{R}\right)$. 
Let $\lambda$ be an affine parameter along this null geodesic with $\lambda=\lambda_{E}$ at the event of emission and $\lambda=\lambda_{R}$ at the event of reception. If we write the line element in the form $d s^{2}=g_{00}(r, \theta, \varphi) d t^{2}-g_{j k}(r, \theta, \varphi) d x^{j} d x^{k}$, then, since the geodesic is null, we must have

$$
g_{00}(r, \theta, \varphi)\left(\frac{d t}{d \lambda}\right)^{2}=g_{j k}(r, \theta, \varphi) \frac{d x^{j}}{d \lambda} \frac{d x^{k}}{d \lambda}
$$

so we can write

$$
\frac{d t}{d \lambda}=\left[\frac{g_{j k}(r, \theta, \varphi)}{g_{00}(r, \theta, \varphi)} \frac{d x^{j}}{d \lambda} \frac{d x^{k}}{d \lambda}\right]^{\frac{1}{2}} .
$$

On integrating between $\lambda=\lambda_{E}$ and $\lambda=\lambda_{R}$ we have

$$
t_{R}-t_{E}=\int\left[\frac{g_{j k}(r, \theta, \varphi)}{g_{00}(r, \theta, \varphi)} \frac{d x^{j}}{d \lambda} \frac{d x^{k}}{d \lambda}\right]^{\frac{1}{2}} d \lambda .
$$

Because the integral on the right-hand side of the above equation depends only on the light path through space, and since the emitter and observer are at fixed positions in space, then $t_{R}-t_{E}$ has the same value for all signals sent. This implies that for any two signals emmited at coordinate times $t_{E}^{(1)}, t_{E}^{(2)}$ and received at $t_{R}^{(1)}, t_{R}^{(2)}$, we have $t_{R}^{(1)}-$ $t_{E}^{(1)}=t_{R}^{(2)}-t_{E}^{(2)}$, which means that the coordinate time difference $\Delta t_{E}=t_{E}^{(2)}-t_{E}^{(1)}$ at the event of emission is equal to the coordinate time difference $\Delta t_{R}=t_{R}^{(2)}-t_{R}^{(1)}$ at the event of reception. On the other hand, we know from Section 3 that the proper time recorded by clocks in a general Weyl frame must be calculated by using the formula

$$
\Delta \tau=\int_{a}^{b} e^{-\frac{\phi}{2}}\left(g_{\mu \nu} \frac{d x^{\mu}}{d \lambda} \frac{d x^{\nu}}{d \lambda}\right)^{\frac{1}{2}} d \lambda .
$$

Therefore, the proper time recorded by the clocks of observers situated at the body and at the point of reception will be given, by the

$$
\Delta \tau_{E}=e^{-\frac{\phi_{E}}{2}} \sqrt{g_{00}\left(r_{E}, \theta_{E}, \varphi_{E}\right)} \Delta t_{E},
$$

and

$$
\Delta \tau_{R}=e^{-\frac{\phi_{R}}{2}} \sqrt{g_{00}\left(r_{R}, \theta_{R}, \varphi_{R}\right)} \Delta t_{R}
$$

where $\phi_{E}=\phi\left(r_{E}, \theta_{E}, \varphi_{E}\right)$ and $\phi_{R}=\phi\left(r_{R}, \theta_{R}, \varphi_{R}\right)$. Since $\Delta t_{E}=\Delta t_{R}$, we have

$$
\frac{\Delta \tau_{R}}{\Delta \tau_{E}}=\frac{e^{-\frac{\phi_{R}}{2}} \sqrt{g_{00}\left(r_{R}, \theta_{R}, \varphi_{R}\right)}}{e^{-\frac{\phi_{E}}{2}} \sqrt{g_{00}\left(r_{E}, \theta_{E}, \varphi_{E}\right)}} .
$$

Suppose now that $n$ waves of frequency $\nu_{E}$ are emitted in proper time $\Delta \tau_{E}$ from an atom situated on the body. Then $\nu_{E}=\frac{n}{\Delta \tau_{E}}$ is the proper frequency measured by an observer situated at the body. On the other hand, the observer situated at the fixed point $\left(r_{R}, \theta_{R}, \varphi_{R}\right)$ will see these $n$ waves in a proper time $\Delta \tau_{R}$, hence will measure a frequency $\nu_{R}=\frac{n}{\Delta \tau_{R}}$. Therefore, we have

$$
\frac{\nu_{R}}{\nu_{E}}=\frac{e^{-\frac{\phi_{E}}{2}} \sqrt{g_{00}\left(r_{E}, \theta_{E}, \varphi_{E}\right)}}{e^{-\frac{\phi_{R}}{2}} \sqrt{g_{00}\left(r_{R}, \theta_{R}, \varphi_{R}\right)}} .
$$


We, thus, see that $\nu_{R} \neq \nu_{E}$, i.e. the observed frequency differs from the frequency measured at the body, and this constitutes the spectral shift effect in a general Weyl frame.

To conclude, two points related to the above equation are worth noting. The first is that since in a Riemann frame $\phi=0$ the Eq. (57) reduces the well-known general relativistic formula for the gravitational spectral shift. The second point is that if we go to a Weyl frame where $g_{00}$ is constant, then Eq. (57) becomes simply

$$
\frac{\nu_{R}}{\nu_{E}}=e^{\frac{1}{2}\left(\phi_{R}-\phi_{E}\right)}
$$

As we see, in this frame all information concerning the gravitational field is contained in the Weyl scalar field.

\section{WIST theory viewed in the Riemann frame}

In Section 2, we have briefly commented on the close correspondence between the mathematical structure of Weyl integral geometry and Riemmanian geometry. More precisely, we have shown that to each Weyl frame $(M, g, \phi)$ there corresponds a unique Riemann frame $\left(M, \widehat{g}=e^{-\phi} g, 0\right)$, such that geometrical objects constructed from $g$ and $\phi$ in the frame $(M, g, \phi)$, such as the affine connection coefficients, curvature, geodesics, etc, can be carried over to $(M, \widehat{g}, 0)$ without ambiguity, and vice-versa. This fact makes us wonder how some gravity theories formulated in a Weyl integral space-time would then appear when viewed in the Riemann frame $\left(M, \widehat{g}=e^{-\phi} g, 0\right)$. A good representative of these theories, in which we would like to focus our attention now, is a proposal known as the Weyl integrable space-time (WIST) [8]. Let us recall the basic tenets of this theory.

The WIST approach starts by postulating the action

$$
S=\int d^{4} x \sqrt{-g}\left\{R+\omega \phi^{, \mu} \phi_{, \mu}+e^{-2 \phi} L_{m}\right\}
$$

where $R$ denotes the Weylian curvature, $\phi$ is the scalar Weyl field, $\omega$ is a dimensionless parameter and $L_{m}$ is the Lagrangian of the matter fields. It is also postulated that the form of $L_{m}$ is obtained from the corresponding Lagrangian in special relativity by substituting simple derivatives by covariant derivatives with respect to the Weyl connection. As regards to the above action, two comments are in order. The first is that it is not invariant under the Weyl transformations (21) and (44). The second, as we shall show now, is that when we go to the Riemann frame $\left(M, \widehat{g}=e^{-\phi} g, 0\right)$ through the Weyl transformations $\widehat{g}_{\mu \nu}=e^{-\phi} g_{\mu \nu}, \widehat{\phi}=\phi-\phi=0$, then (158) becomes

$$
S=\int d^{4} x \sqrt{-\widehat{g}} e^{\phi}\left\{\widehat{R}+\omega \widehat{g}^{\mu \nu} \phi_{, \mu} \phi_{, \nu}+L_{m}\right\}
$$

where by $\widehat{R}$ we are denoting the scalar curvature defined in terms of $\widehat{g}_{\mu \nu}$. Changing to the field variable $\Phi=e^{\phi}$, we finally get

$$
S=\int d^{4} x \sqrt{-\widehat{g}}\left\{\Phi \widehat{R}+\frac{\omega}{\Phi} \widehat{g}^{\mu \nu} \Phi_{, \mu} \Phi_{, \nu}+L_{m}\right\}
$$


which we immediately recognise as the action of Brans-Dicke theory of gravity written in units such that $\frac{8 \pi}{c^{4}}=1[12$. We, thus, see that in the Riemann frame the WIST action (58) is formally identical to the Brans-Dicke action (60), where $\Phi$ is no longer interpreted as a geometrical field. This reminds us of a similar situation in which Brans-Dicke theory is interpreted in two different frames, the Jordan and Einstein frames, an issue widely discussed in the literature [24].

The mathematical analogy between WIST and Brans-Dicke theories works in both directions. Thus, one may start the action (60), which gives Brans-Dicke theory in the usual Riemannian (Jordan) frame, and then go to the Weyl frame (Einstein frame) in which the action takes the form of (58), where the scalar field $\phi$ might be interpreted as a geometric field. The usual view, let us say, the non-geometrical view, is that we have the same Brans-Dicke theory in two different frames, the Jordan and Einstein frame. The physical interpretation of the two pictures has been widely discussed in the literature [24]. However, a characteristic feature of Brans-Dicke theory is that Newton's gravitational constant $G$ is replaced by the inverse of the scalar field, i.e. $G=\Phi^{-1}$, an idea that goes back to Dirac [20]. Similarly to the original Weyl theory, which represents an elegant way of geometrizing the electromagnetic field [3], the same can be said of the WIST theory as regards to the scalar field: we have here a geometrization of a scalar field. In view of this analogy, the passage from the Jordan frame to the Einstein frame may be interpreted as a "geometrization" of $G$, the empirical physical quantity that sets the strength of the gravitational force, now promoted to the status of a field. One may perhaps feel inclined to regard this geometrical attempt to explain the origin of $G$ as being in accordance with the Machian view that local physical laws are determined by the large-scale structure (geometry) of the universe [26].

It is worth noting that a connection between Brans-Dicke theory and Weyl integrable geometry appears in a different context. In fact, this connection has been proved to exist for any scalar-tensor theory in which the scalar field is non-minimally coupled to the metric [27, 11]. Without going into the details, the argument is the following. We start with the action (59) in the absence of matter and consider variations in the sense of Palatini approach, i.e. treating the metric and the affine connection separately as dynamical variables. It is then not difficult to show that the variation with respect to the connection leads to the equation (1), that is, the compatibility condition that defines a Weyl integrable manifold.

\section{Final remarks}

As we have seen, it is possible to set up a different scenario of general relativity theory in which the gravitational field is not associated with the metric tensor only, but with the combination of both the metric $g_{\mu \nu}$ and a geometrical scalar field $\phi$. In this scenario we have a new kind of invariance and the same physical phenomena may appear in different pictures and distinct representations. This can be well illustrated when we consider, for instance, homogeneous and isotropic cosmological models. All these have a conformallyflat geometry, and as a consequence, there is a frame in which the geometry of these models becomes that of flat Minkowski space-time. In the Riemann frame the spacetime manifold is endowed with a metric that leads to Riemannian curvature, while in the Weyl frame space-time is flat. In this case, all information about the gravitational field is 
encoded in the scalar field. Another example is given by the gravitational spectral shift, in which the Weyl scalar field plays an essential role.

The presence of a scalar field in an arbitrary Weyl frame also leads to formal analogy with Brans-Dicke theory, a fact that has already been known and mentioned in the literature [14]. Because of this $\mathrm{O}^{\prime}$ Hanlon-Tupper space-time in Brans-Dicke theory with $\omega=-\frac{3}{2}$ can be regarded as Minkowski space-time in a Weyl frame, although the analogy is not perfect since in Brans-Dicke theory test particles follow metric geodesics rather than affine Weyl geodesics.

An important conclusion to be drawn from what has been presented in this paper is that general relativity can perfectly "survive" in a non-Riemannian environment. Moreover, as far as physical observations are concerned, all Weyl frames, each one determining a specific geometry, are completely equivalent. In a certain sense, this would reminds us of the view conceived by $\mathrm{H}$. Poincaré that the geometry of space-time is perhaps a convention that can be freely chosen by the theoretician [28]. In particular, according to this view, general relativity might be rewritten in terms an arbitrary conventional geometry [29].

Finally, we should also note that the same formalism we have used to recast general relativity in a form that is manifestly invariant under Weyl transformations may be extended in a straightforward way to the so-called $f(R)$ theories [30, where the issue of physical interpretation between the Einstein and Jordan frames may be of interest [31]. The basic idea here is to start with the action $S=\int d^{4} x \sqrt{-g}\left\{f(R)+\kappa L_{m}(g, \xi)\right\}$, where $\xi$ stands generically for the matter fields. We then follow the same procedure presented in Section 3 and postulate that this action may be regarded as defined in a Weyl integral space-time in a particular frame where the Weyl scalar field vanishes, that is, in the Riemann frame. The next step is almost obvious: using the fact that the combination $e^{\phi} R$ is invariant under (2) and (4) the sought-after action in an arbitrary Weyl frame will be given by $S=\int d^{4} x \sqrt{-g} e^{-2 \phi}\left\{f\left(e^{\phi} R\right)+\kappa L_{m}(g, \xi)\right\}$, where for the definition of $L_{m}(g, \xi)$ in an arbitrary frame the prescriptions outlined in Section 3 still apply. We leave the details of this extension for a separate publication.

\section{Acknowledgments}

C. Romero and M. L. Pucheu would like to thank CNPq/CLAF for financial support. We are grateful to Dr. I. Lobo for helpful discussions and suggestions.

\section{References}

\section{References}

[1] P. Dirac, Proc. Roy. Soc. London A 333 , 403 (1973).

[2] J. D. Norton, Rep. Prog. Phys. 56, 791 (1993).

[3] H. Weyl, Sitzungesber Deutsch. Akad. Wiss. Berli 465 (1918); H. Weyl, Space, Time, Matter (Dover, New York, 1952).

[4] H. Goenner, Living Rev. Rel. 7, 2 (2004). 
[5] A nice account of Weyl's ideas as well as the refutation of his gravitational theory may be found in W. Pauli, Theory of Relativity (Dover, New York, 1981). See, also, L. O'Raiefeartaigh and N. Straumann, Rev. Mod. Phys. 72, 1 (2000). For a more formal mathematical treatment, see G. B. Folland, J. Diff. Geom. 4, 145 (1970).

[6] See, for instance, B. Schuz, Geometrical methods of Mathematical Physics, Ch.2 ( Cambridge University Press, 1980).

[7] A. Pais, Subtle is the Lord (Oxford University Press, 1983)

[8] M. Novello and H. Heintzmann, Phys. Lett. A 98, 10 (1983); K. A. Bronnikov, Yu. M. Konstantinov and V. N. Melnikov, Grav. Cosmol. 1, 60 (1995); M. Novello, L.A.R. Oliveira, J.M. Salim and E. Elbas, Int. J. Mod. Phys. D 1, 641 (1993); J. M. Salim and S. L. Sautú, Class. Quant. Grav 13, 353 (1996); H. P. de Oliveira, J. M. Salim and S. L. Sautú, Class. Quant. Grav. 14, 2833 (1997); V. Melnikov, Classical Solutions in Multidimensional Cosmology in Proceedings of the VIII Brazilian School of Cosmology and Gravitation II, ed. M. Novello (Editions Frontières, 1995) p.542; R. G. Gannouji, H. Nandan, N. Dadhich, JCAP 11, 51 (2011).

[9] O. Arias, R. Cardenas and I.Quiros, Nucl. Phys. B 643, 187 (2002); J. Miritzis, Class. Quant .Grav. 21, 3043 (2004); J. Miritzis, J.Phys.: Conf. Ser. 8, 131 (2005); M. Israelit, Found. Phys. 35, 1725 (2005); F. Dahia, G. A. T. Gomez and C. Romero, J. Math.Phys. 49, 102501 (2008); J. E. Madriz Aguilar and C. Romero, Found. Phys. 39, 1205 (2009). T. Moon, J. Lee, P. Oh, Mod. Phys. Lett. A 25, 3129 (2010), arXiv.gr-qc/0912.0432.

[10] S. R. Mainwaring and G. E. Stedman, Phys. Rev. A 47, 3611 (1993).

[11] F. P. Poulis and J. M. Salim, Int. J. Mod. Phys.: Conf. Series, 3, 87 (2011), arXiv:grqc/1106.3031.

[12] C. H. Brans and R. H. Dicke, Phys. Rev. 124, 925 (1961). R. H. Dicke, Phys. Rev. 125, 2163 (1962).

[13] R. Adler, M. Bazin and M. Schiffer, Introduction to General Relativity (McGraw-Hill, 1975).

[14] Canuto, P. J. Adams, S. H. Hsieh, E. Tsiang, Phys. Rev. D 16, 1643 (1977). N. Deruelle and M. Sasaki, arXiv:gr-qc/1007.3563 (2010). D. Blaschke and M. Dabrowski, hep-th/0407078. J. C. Fabris, S.V. B. Gonçalves and R. S. Ribeiro, Gravit. Cosmol. 12, 49 (2006) [astro-ph/0510779].

[15] M. P. Dabrowski, T. Denkiewicz and D. Blaschke, Annalen Phys. 16, 237 (2007).

[16] J. O‘Hanlon and B. Tupper, Nuovo Cimento B 7, 305 (1972).

[17] J. P. Mimoso and D. Wands, Phys. Rev. D 51, 477.

[18] C. Romero and A. Barros, Phys. Lett. A 173, 243 (1993). F. M. Paiva and C. Romero, Gen. Rel .Grav. 25, 1305 (1993). 
[19] V. Faraoni, Cosmology in Scalar-Tensor Gravity (Kluwer Academic Publishers, Dordrecht, 2004).

[20] P. A. M. Dirac, Nature, 139, 323 (1937).

[21] R. T. Singh and D. Shridhar, Int. J. Theor. Phys. 26, 901 (1987).

[22] C. Romero, J. B. Fonseca-Neto and M. L. Pucheu, Found. Phys. (to appear), arXiv: gr-qc/1101.5333.

[23] M. Ibison, J. Math. Phys 48, 122501 (2007).

[24] V. Faraoni, Int. J. Theor. Phys, 38, 217 (1999). V. Faraoni, E. Gunzig and P. Nardoni, Fund. Cosm. Phys. 20, 121 (1999). V. Faraoni and S. Nadeau, Phys. Rev. D 75, 23501 (2007).

[25] I. Quiros, R. Bonal and R. Cardenas, Phys. Rev. D 62, 044042 (2000). I. Quiros, R. Garxía-Salcedo and J. E. Madriz Aguilar, arXiv: gr-qc/1108.2911v2 (2011). I. Quiros, R. Garxía-Salcedo, J. E. Madriz Aguilar and T. Matos, arXiv: gr-qc/1108.5857v2 (2011).

[26] S. W. Hawking and G. F. R. Ellis, The Large Scale Structure of Space-Time (Cambridge University Press, 1973).

[27] See, for instance, M. Novello, "Theoretical Cosmology", in VII Brazilian School of Cosmology and Gravitation, Ed. M. Novello (Editions Frontières, 1995).

[28] H. Poincaré, Science and Hypothesis (Dover, New York, 1952).

[29] I. W. Roxburgh and R. K. Tavakol, Found. Phys. 8, 229 (1978).

[30] For a nice review on $f(R)$ theories, see T. P. Sotiriou and V. Faraoni, Rev. Mod. Phys. 82, 451 (2010).

[31] Iain A. Brown and A. Hammami, arXiv: gr-qc/1112.0575v2 (2011). 